\title{
Kernos
}

Revue internationale et pluridisciplinaire de religion grecque antique

15 | 2002

Varia

\section{Susan DEACY, Alexandra VILLING (éds), Athena in the Classical World}

\section{Catherine Lecomte}

\section{OpenEdition \\ Journals}

\section{Édition électronique}

URL : http://journals.openedition.org/kernos/1428

DOI : $10.4000 /$ kernos. 1428

ISSN : 2034-7871

\section{Éditeur}

Centre international d'étude de la religion grecque antique

Édition imprimée

Date de publication : 1 janvier 2002

ISSN : 0776-3824

\section{Référence électronique}

Catherine Lecomte, "Susan dEACY, Alexandra viLuINg (éds), Athena in the Classical World », Kernos [En ligne], 15 | 2002, mis en ligne le 16 juin 2011, consulté le 21 septembre 2020. URL : http:// journals.openedition.org/kernos/1428; DOI : https://doi.org/10.4000/kernos.1428 
their relation to the rituals performed at Brauron, while Petros Themelis focused on the topography of the sanctuary in the light of a 3 rd-century- $\mathrm{BC}$ inscription found at Brauron, which mentions a number of buildings. Mario Guiman explored the krokotos, the signification of the colour of this piece of clothing in antiquity and the plant from which the colouring was derived, the crocus. Dina Peppas-Delmousou commented on the sacred money of Apollon mentioned in a stele from 416/15 BC found at Brauron and the presence of a strong male aspect at the sanctuary, illustrated by a 4th-century dedication by some stratiotai on behalf of their commander, perhaps after having completed their military service. The seminar at Urbino clearly must have been a very interesting event but one would have wished for some information on where the contributions of the wellknown participants will be published in full.

More related to the sphere of feminine cults in general rather than to Brauron is the contribution by Saviero Gualerzi, dealing with a number of questions concerning Sappho and her female thiasos on Lesbos. The author provides a sort of Stand der Forscbung on Sappho and her contemporary environment and discusses how participation in this tbiasos may have functioned as a preparation for the future marriages of the young women, in particular pertaining to the emotional aspects, but also physical beauty and sexuality. Of central interest is also the role of homosexual love within Sappho's group, a matter on which scholars' opinions are divided. Wilamowitz, for example, claimed that the thiasos was only a religious group concerned with the cult of Aphrodite and the poetry of Sappho, an interpretation which Gualerzi finds to be lacking in imagination and neatly remarks upon as la visione poco elastica dell'autore tedesco (p. 49).

On the whole, this is more a book about scholarship than actual research focusing on Brauron and female cults. For whom this work is conceived is not entirely clear but it is best seen as an introduction to the sources and research concerning Brauron in particular and female cults in general. One dominating aspect is the review of scholarship. Here, however, one feels a certain tendency of bias towards Italian and French research at the cost of the general picture and this is far from constituting an attempt at an overall bibliography. There is, for example, no reference to Ken Dowden's Death and the maiden, a study which is just as important for Brauron as Pierre Brulé's La fille d'Athènes. The book could function as an introduction to the whole Brauron complex for students but if this was one of the intentions, it might have been achieved in a more comprehensive manner.

Gunnel Ekroth

(Stockholm University)

Susan Deacy, Alexandra Villing (éds), Athena in the Classical World, Leiden, Brill, 2001. 1 vol. $16 \times 24,5 \mathrm{~cm}$, xII +435 p., 24 pl. ISBN : 90-0412-142-0.

Ce volume rassemble une vingtaine de communications faites lors du colloque sur Athéna organisé au Lindon College d'Oxford en avril 1998. Les auteurs ambitionnent à la fois de combler un vide dans les études de religion grecque, dont aucune n'a été récemment consacrée à la déesse ( $c f$. l'état de la question fourni par les éditrices en introduction), et de proposer une approche interdisciplinaire du sujet. Les articles sont répartis en trois catégories, quelque peu artificielles car elles se recoupent continuellement : le culte, le rituel et le mythe; les représentations artistiques; les traitements littéraires. On obtient ainsi un livre extrêmement riche et diversifié, dont le titre ne recouvre cependant pas exactement le contenu, puisque la Grèce occupe une place écrasante dans ce "monde classique ", tandis que l'Italie ne suscite que deux articles (Athéna dans l'Énéide 
et dans son rapport avec Minerve), que l'on s'aventure dans l'Anatolie hittite et en Inde à la recherche des origines, et que l'on suit la déesse jusqu'à la fin du $\mathrm{xIX}^{\mathrm{e}}$ S., avec les «Sécessionnistes 》 Franz von Stuck et Gustav Klimt.

Sont d'abord étudiés deux attributs d'Athéna, l'égide ( $N$. Robertson) et la salpinx (A. Serghidou). Chaque année, en septembre, le Palladium était enlevé du temple où il était normalement conservé, près de l'llissos, et transporté, dépourvu de l'égide, au Phalère. En novembre, la statue retrouvait son temple athénien et était revêtue d'une nouvelle égide. Le retour du Palladium était marqué au Pha lère par une double procession, l'une à l'intérieur du port et l'autre vers Athènes, et par un combat rituel entre éphèbes. Ce combat doit très certainement être mis en rapport avec les nombreux récits de lutte pour le Palladium qui circulaient dans le monde grec. Selon Neil Robertson, ce rituel montre qu'Athéna était une "Weather Goddess ». L'égide était en effet obtenue à partir du sacrifice d'une chèvre, animal dont l'apparition dans le ciel (l'étoile "Capella » ou "Chèvre ", dans la constellation du Cocher) en septembre annonçait de violents orages. Pour se protéger des intempéries au moment des labours et des semailles, les Athéniens rendaient le Palladium inefficace en lui enlevant son égide et en lui faisant quitter la ville; trois mois plus tard, lorsque la pluie était nécessaire pour faire pousser les récoltes, ils se le réappropriaient.

La salpinx, comme l'aulos, montrent l'intérêt d'Athéna pour la musique. Mais ils témoignent aussi de l'idéologie politique qui sous-tend leur utilisation par la déesse. Ce n'est pas seulement parce qu'il la défigure qu'Athéna rejette l'aulos, mais aussi parce que cet instrument, qui passait pour être d'origine asiatique et dont jouaient les esclaves, n'était pas digne d'un homme libre. La trompette, au contraire, annonçait la guerre et l'ouverture des assemblées; elle reflétait donc parfaitement le double rôle guerrier et politique de la déesse, qu'elle porte l'épiclèse Polias à Athènes ou Salpinx à Argos.

De nombreux articles mettent en évidence l'utilisation politique qui peut être faite d'une figure divine. L'Athéna casquée - car c'est bien d'elle qu'il s'agit, et non d'Aphrodite, comme le montre S. Ritter - doit sa présence sur les monnaies de Corinthe à la volonté de la ville d'exprimer une alternative aux représentations de la déesse sur le numéraire de l'autre grande puissance de l'époque, Athènes. L'Athéna Nicéphore des monnaies de Pergame, dont l'iconographie présente des caractères à la fois grecs et orientaux, fut choisie comme patronne de l'alliance économique qui fut scellée, à l'occasion de la victoire d'Eumène II sur les Galates, entre Pergame et six autres cités, dont certaines, comme Éphèse et Sardes, abritaient des cultes orientaux (A.S. Faita). Il est à peine besoin de rappeler, à la suite de Jenifer Neils, le puissant message politique que véhicule l'association de Zeus et d'Athéna dans la cité athénienne. Sarah Spence fournit sans doute le plus bel exemple d'interaction entre mythe, culte, iconographie et littérature. Elle pose la question du rôle respectif d'Athéna et d'Apollon dans l'idéologie augustéenne, à travers deux documents, l'un littéraire et l'autre figuré : l'Énéide, et un cratère fragmentaire qui met en parallèle le héros troyen sacrifiant à Athéna et un personnage, perdu mais qui est très certainement Auguste, sacrifiant à Apollon. On voit ainsi qu'Auguste, au moment où il introduit les dieux au Palatin et les installe dans le nouveau temple d'Apollon, se compare à Énée introduisant les dieux dans le Latium et les installant dans le temple de Minerve qu'il vient de construire.

Les données archéologiques et iconographiques véhiculent évidemment des informations fondamentales. $\AA$ Stymphale, les restes du sanctuaire de l'acropole, en particulier les offrandes votives, semblent confirmer une inscription qui attribue le culte à Athéna Polias ( $\mathrm{H}$. Williams - G. Schaus). Les plaques et plats en 
terre cuite trouvés sur l'acropole d'Athènes illustrent, mieux que tout autre support, combien l'iconographie des offrandes est adaptée à la divinité à laquelle elles sont destinées (C. Wagner). Les représentations figurées évoluent également avec le temps et les sociétés qui les produisent. Ainsi, à la fin de l'époque classique et à l'époque hellénistique, les statuettes d'Athéna, qui donnent à la déesse une apparence presque enfantine, correspondent-elles à un moindre rôle religieux et politique de la déesse au cœur de la cité (I. Altripp). Dans l'Alexandrie hellénistique, le type de l'Athéna Velletri se transforme en une Athéna Tyché protectrice des Ptolémées (E. Mathiopoulos). La déesse que l'on montre entièrement armée, à l'époque de la menace perse, se transforme en une figure de plus en plus érotique, comme pour transposer en image les paroles de Périclès invitant ses concitoyens à aimer leur ville comme on tombe amoureux d'une femme (très bel article de L. LLewellyn-Jones sur la sémiotique du vêtement).

L'image d'Athéna évolue également dans la littérature. On rit de la déesse dans la Batrachomyomachie, comme on ne l'aurait jamais fait dans l'Odyssée ni même dans la comédie ( $S$. Milanezi). A. Karanika étudie l'influence des poèmes homériques sur les pratiques cultuelles athéniennes du $v^{e} s$. T. Papadopoulou détaille les apparitions d'Athéna dans la tragédie. L'article de C. Higbie sur la Chronique de Lindos fournit un exemple remarquable de réélaboration «littéraire » du mythe. Les Rhodiens ont en effet comblé les "trous » de l'épopée homérique en imaginant que des héros de la guerre de Troie avaient fait escale sur leur île pour apporter à Athéna Lindia des offrandes dont la Chronique fournit une liste. Le prestige de la déesse s'en trouvait ainsi augmenté, de même que celui des Rhodiens, qui se rattachaient de la sorte au glorieux passé mythique grec.

Fritz Graf propose une analyse particulièrement intéressante, notamment du point de vue méthodologique. Il pose la question de savoir ce qui a permis l'équation Athéna-Minerve et quelles furent les conséquences de cette identification sur le culte de Minerve. Il apparaît que les caractères propres à la déesse italienne ont été complètement occultés par son identification précoce avec l'Athéna grecque, dont elle adopte l'iconographie dès l'époque archaïque, et dont les fonctions recouvrent les siennes, à l'exception peut-être du domaine médical.

On signalera enfin les articles de A. Teffeteller et de N. Allen, qui clôturent le volume par deux hypothèses sur les origines d'Athéna. La première suggère qu'Athéna aurait hérité son nom et ses fonctions guerrière et poliade de la grande déesse anatolienne de la guerre, la déesse solaire d'Arinna - les rapprochements que l'on peut établir entre les deux figures semblent cependant quelque peu superficiels : leur lien à la guerre et leur fonction de protectrices du roi. Plus convaincante, la comparaison que $N$. Allen établit entre l'Athéna de l'Odyssée et la Durgâ du Mabâbhârata indique selon toute probabilité que la trame des deux épopées et les rôles parallèles qu'y jouent les déesses remontent à une même "proto-narration » indo-européenne.

Au total, cette Athena in the Classical World fournit effectivement une très intéressante présentation interdisciplinaire de différents aspects de la personnalité d'Athéna. Parmi les enseignements de ce volume, on retiendra, comme le montrent de nombreux articles, que la façon dont les Athéniens perçoivent et représentent leur déesse poliade n'est pas celle de l'ensemble du monde grec, et qu'il faut donc éviter de recourir aux standards athéniens comme à des valeurs panhelléniques. Une bibliographie de plusieurs pages, regroupant principalement les recherches récentes sur Athéna, permettra d'approfondir les sujets abordés dans 
le livre, dont plusieurs index détaillés rendent la consultation particulièrement aisée.

Catherine Lecomte (Université de Liège)

Sabina Mazzoldi, Cassandra, la vergine e l'indovina. Identità di un personaggio da Omero all'Ellenismo, Pisa/Roma, Istituti editoriali e poligrafici internazionali, 2001. 1 vol. $14,5 \times 22,5 \mathrm{~cm}, 336$ p., 21 pl. ISBN : 888147-244-9.

Ces dernières années, le personnage de Cassandre, ainsi que le monde de la prophétie antique en général, ont connu un intérêt croissant, justifié par l'importance de cet aspect dans la religion et la littérature grecques. Le livre classique de J. Davreux (La légende de la prophétesse Cassandre d'après les textes et les monuments, Liège, 1942), encore utile, n'était pas du tout satisfaisant dans le traitement de certaines questions et, en ce qui concerne la partie iconographique, devait être mis à jour. Si le LIMC a contribué à remplir la dernière lacune, les études de D. Neblung (Die Gestalt der Kassandra in der antiken Literatur, Suttgart/Leipzig, 1997) et celle qui est maintenant le sujet de ce compte rendu ont satisfait géné reusement les exigences de révision des questions littéraires, et même (dans le cas de l'ouvrage de Mazzoldi) de l'étude des images.

Pour ceux qui pourraient se poser la question de la justification de cet ouvrage après la parution de celui de Neblung, je donne la parole à l'A. : «Scopo del lavoro non è fornire un repertorio delle fonti classiche (...) ma studiare le valenze del personaggio attraverso un approfondimento filologico e letterario di quelle testimonianze nelle quali la tipologia di Cassandra si definisce e si consolida, a partire da Omero fino alla letteratura ellenistica ». Il y a donc une différence d'extension chronologique et une autre, importante, dans l'analyse littéraire et linguistique des témoignages. Ce dernier trait est le plus remarquable du présent ouvrage et fait de lui un très utile instrument pour l'interprétation des textes étudiés (ce qui ne surprend pas, vu qu'il s'agit d'une thèse de doctorat dirigée par Paola A. Bernardini à Urbino).

Après la «Premessa » (p. 11-12) et l'Introduction (p. 13-21), le livre est divisé en deux parties. La première est une analyse approfondie du motif de la virginité

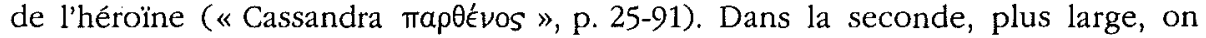
étudie les témoignages littéraires et les traits du langage prophétique qui caracté rise ces prédictions ( «Cassandra $\mu a ́ \nu T \iota s », p .93-283$ ). On ajoute un Appendice avec le lexique mantique (c'est-à-dire, «les mots les plụs significatifs employés par Cassandre ou en rapport avec elle » [p. 285, n. 1], p. 285-290). Suivent une excellente bibliographie (p. 291-318), un index des sources iconographiques, classé par thèmes (p. 321-330), un autre de noms mythiques et de notabilia (331334 ) et un dernier des passages discutés (p. 335-336). L'ouvrage se referme sur les photographies de 25 représentations figurées. L'édition est bien soignée et presque sans 'coquilles'.

La question de la virginité est analysée en trois chapitres : le premier a un caractère plus général, le deuxième traite le mythe du sacrilège d'Ajax et le troisième les rapports entre Cassandre et Agamemnon. Le premier ( Il yáfos mancato ", p. 27-30) établit bien que, depuis les sources les plus anciennes, le mariage de l'héroïne est un 'inaccomplissement' permanent. Elle est toujours 'projetée' vers ce but, mais elle n'y réussit jamais. En d'autre mots, Cassandre, en tant que femme, est définie négativement, presque comme une 'non-femme'. La lecture du deuxième chapitre ("Cassandra, Aiace e lo Góavov di Atena », p. 31-61) 